\title{
Proteases are Modulated by Fascin in Oral Cancer Invasion
}

\author{
Min Kyeong Lee, Ji Hyeon Park, Seol Hwa Gi, Young Sun Hwang \\ Department of Dental Hygiene, College of Health Science, Eulji University, Seongnam, Korea
}

\begin{abstract}
Background: Cancer invasion is a critical factor for survival and prognosis of patients with cancer. Identifying and targeting factors that influence cancer invasion are an important strategy to overcome cancer. In this study, we investigated the role of fascin known to be associated with cancer invasion.

Methods: Fascin depletion was performed with lentiviral short hairpin RNA against fascin mRNA and stable cell line (Fascin ${ }^{\text {dep }}$ ) was established. Matrigel-Transwell invasion and three-dimensional (3D) culture system were used to observe fascin depletion effects. In order to observe the changes of protease secretion by fascin depleted cancer cells, protease antibody array was performed.

Results: Fascin was highly expressed in invasive cancer cells. Fascin-depleted cells showed decreased cancer invasion in Matrigel-Transwell invasion and 3D culture system. In addition, inhibition of proteases secreation and decrease of intracellular proteases mRNA expression were observed in fascin deplete cells.

Conclusions: These results indicates that fascin is closely involved in proteases activity and cancer invasion. Therefore, fascin is a strategically important factor for controlling cancer invasion.
\end{abstract}

(J Cancer Prev 2018;23:141-146)

Key Words: Metalloproteases, Fascin, Head and neck neoplasms, Gene silencing, Tumor microenvironment

\section{INTRODUCTION}

Cancer growth and invasiveness are closely related to cancer progression and prognosis. ${ }^{1}$ Malignant tumors are highly related to poor survival rate of cancer patients. The incidence of oral cancer varies among races and living environments. Oral cancer is one of the 10 most common cancer in the world, accounting for $3 \%$ to $5 \%$ of total carcinoma in the human body. Oral squamous cell carcinoma (OSCC) is the most common malignant cancer. Like other types of cancer, oral cancer can be restored $80 \%$ to $90 \%$ in early detection. However, more than half of the patients are diagnosed after the cancer has progressed considerably, leading to poor prognosis. ${ }^{2}$ Despite extensive studies and improvements in the treatment of OSCC, the 5-year survival rate is only $53 \%$, which is unchanged over the past 40 years. ${ }^{3}$
Because local recurrence and metastasis to distance organs of oral cancer occur frequently, treatment strategies to reduce or inhibit local recurrence are appropriate to improve the survival rate of patients with oral cancer. Therefore, finding diagnostic factors with high predictive accuracy for cancer invasion and metastasis is important for cancer prognosis. However, oral tissue is composed of heterogenous cells such as teeth, bone, and soft tissues. The location of cancer development also varies, making it difficult to find a suitable diagnostic marker for oral cancer invasion and metastasis.

Invasive cancer cells are known to form a protrusive cellular structure called invadopodia to infiltrate the surrounding matrix and the basement membrane. ${ }^{4}$ When invasion is needed, cancer cells induce actin polymerization based on Arp2/3 complex and neuronal Wiskott-Aldrich syndrome protein (N-WASP) to

Received August 31, 2018, Revised September 13, 2018, Accepted September 19, 2018

Correspondence to: Young Sun Hwang

Department of Dental Hygiene, College of Health Science, Eulji University, 553 Sanseong-daero, Sujeong-gu, Seongnam 13135, Korea

Tel: +82-31-740-7493, Fax: +82-31-740-7352, E-mail: kiteys@eulji.ac.kr, ORCID: Young Sun Hwang, https://orcid.org/0000-0001-7012-3434

Copyright (C) 2018 Korean Society of Cancer Prevention

(c) This is an Open Access article distributed under the terms of the Creative Commons Attribution Non-Commercial License (http://creativecommons.org/icenses/by-nc/4.0) which permits unrestricted non-commercial use, distribution, and reproduction in any medium, provided the original work is properly cited. 
stabilize the protruding structure by joining many molecules, such as Tsk5, cortactin, synaptjanin-2, Abl-family kinase Arg4, and integrin. Upon completion of the assembly of invadopodia, proteases are secreted and invasion proceeds. Since inhibition of functional invadopodia formation can effectively inhibit cancer invasion, invadopodia-targeted cancer suppression strategies have been suggested to be very effective. In the present study, we observed changes in cancer invasion induced by fascin, a component of functional invadopodia, and derived related proteases.

\section{MATERIALS AND METHODS}

\section{Cell culture}

YD-10B, HSC-2, HSC-3, Ca9.22 OSCC cells were obtained from the Yonsei University College of Dentistry, Republic of Korea, and all were cultured in Dulbecco's Modified Eagle's Medium (DMEM)/F12 (3 : 1 ratio) medium supplemented with 10\% FBS, $1 \times$ $10^{-10} \mathrm{M}$ cholera toxin, $0.4 \mathrm{mg} / \mathrm{mL}$ hydrocortisone, $5 \mu \mathrm{g} / \mathrm{mL}$ insulin, $5 \mu \mathrm{g} / \mathrm{mL}$ apo-transferrin, and $2 \times 10^{-11} \mathrm{M}$ triiodothronine (T3) in a humidified atmosphere of $5 \% \mathrm{CO}_{2}$ at $37^{\circ} \mathrm{C}$. PC-3 prostate cancer cells were cultured in RPMI 1640 medium with 10\% FBS. To establish fascin-depleted YD-10B cell line, fascin 1-specific short hairpin RNA (shRNA) (h) lentiviral particles were transduced in cultured cell with $5 \mu \mathrm{g} / \mathrm{mL}$ polybrene according to the manufacturer protocol (Santa Cruz, Santa Cruz, CA, USA). Continual selection was followed with $1 \mathrm{mg} / \mathrm{mL}$ puromycin to establish fascin-depleted stable cell line (Fascin ${ }^{\text {dep}}$ ). Control shRNA (h) lentiviral particles-A (Santa Cruz) was also used as a cell control (Mock). The extent of fascin depletion was evaluated by Western blot.

\section{Reagents}

All reagents used for cell culture were purchased from Gibco BRL Co. (Rockville, MD, USA). Cholera toxin, hydrocortisone, insulin, apo-transferrin, T3, dimethyl sulfoxide (DMSO), and parthenolide were obtained from Sigma-Aldrich (Sigma-Aldrich Chemical Co., St. Louis, MO, USA). The following antibodies were purchased from their respective sources: Fascin (clone 55K-2, monoclonal mouse anti-human) and E-cadherin (clone NCH-38, monoclonal mouse anti-human) (Dako, Carpentaria, CA, USA); $\beta$-actin (clone AC-74) (Sigma-Aldrich Chemical Co.).

\section{Western blotting}

Protein $(50 \mu \mathrm{g})$ were separated on SDS-PAGE gel and were transferred to a polyvinylidene difluoride membrane (Millipore,
Billerica, MA, USA). The membrane was blocked with $10 \%$ skim milk in PBS containing 0.1\% Tween-20 (PBS-T) and subsequently incubated overnight with a $1: 1,000$ dilution of primary antibody against its specific protein at $4^{\circ} \mathrm{C}$. The blots were then incubated with a 1:3,000 dilution of their respective horseradish peroxidase-conjugated secondary antibodies for 2 hours at room temperature and were washed with PBS-T. The targeted proteins were visualized using an enhanced chemiluminescence detection kit (Amersham Life Science, Arlington Heights, IL, USA) according to the manufacturer's instructions.

\section{Invasion assay}

Eight $\mu \mathrm{m}$ pore sized polycarbonate nucleopore filter inserts in a 24-well Transwell chamber (Costar; Corning, Cambridge, MA, USA), were coated with Matrigel (30 $\mu \mathrm{g} /$ well; Becton Dickinson, Lincoln Park, NJ, USA). Cells $\left(5 \times 10^{4}\right.$ cells) were added into the upper chamber, and complete medium was added to the bottom chamber and keep for 48 hours at $37^{\circ} \mathrm{C}$ incubator. Invaded cells on the lower surface of the membrane was fixed with ethanol and noninvasive cells were removed with a cotton swab. Then cells were stained with hematoxylin. Invaded cells from five fields were counted under a microscope.

\section{Three-dimensional invasion}

Dermal equivalent gel was generated with a Type I-A collagen mixture with eight volumes of ice-cold collagen solution (Nitta Gelatin Inc., Osaka, Japan), one volume of $10 \times$ reconstitution solution $\left(0.022 \mathrm{~g} / \mathrm{mL} \mathrm{NaHCO}_{3}, 0.0477 \mathrm{~g} / \mathrm{ml}\right.$ HEPES, $\left.0.05 \mathrm{~N} \mathrm{NaOH}\right)$, and one volume $10 \times$ DMEM. Immortalized gingiva fibroblast $(1 \times$ $10^{5}$ cells) was added to collagen mixture. ${ }^{5}$ This mixture was poured into polycarbonate filter inserts ( $3 \mu \mathrm{m}$ pore size, $12 \mathrm{~mm}$ diameter; Millipore) and placed in 6-well plates (Costar; Corning). After 24 hours incubation at $37^{\circ} \mathrm{C}$, YD-10B cells $\left(1 \times 10^{5}\right.$ cells) were loaded on collagen gel and complete medium was added to the 6-well plates. The culture medium was then changed every 2 to 3 days for 2 weeks. Gels were then formalin-fixed, paraffin-embedded and histologically examined. To measure invasive areas and depth, the culture tissue was stained with H\&E.

\section{Protease array}

Wild type control Mock and Fascin ${ }^{\text {dep }}$ cells were cultured in 1\% medium and harvested after 24 hours. Harvested medium was centrifuged in 3,000 $\times g$ for 10 minutes and used as conditioned media (CM) for protease array. The protein concentration of the CM was normalized by dilution with serum-free media. Then CM 
was incubated for 24 hours with the Proteome Profiler Human Protease Array Kit (R\&D Systems, St. Louis, MO, USA). The relative expression levels of the proteases were determined according to the manufacturer's protocol and signal intensities were compared using ImageJ software program.

\section{Reverse transcription-PCR}

Total RNA was isolated using TRIzol reagent (Life Technologies, Foster City, CA, USA) according to the instructions provided by the manufacturer. Reverse transcription to obtained cDNA and amplification by PCR using specific primer pairs. Primer sequences are given in Table 1 . The reaction products were electrophoresed on $2 \%$ agarose gel. The obtained bands were visualized with Quantity One software and the Gel Doc 2000 system (Bio-Rad Laboratories, Hercules, CA, USA).

\section{Statistical analysis}

The statistical analysis was performed using InStat GraphPad Prism ver. 5.01 statistical software (GraphPad Software, Inc., San Diego, CA, USA). Results are expressed as mean $\pm \mathrm{SD}$. $P$-values $<$ 0.05 were considered significant.

\section{RESULTS}

\section{Effect of fascin on cancer invasion}

Expression of fascin was confirmed in various cancer cell lines. As shown in Figure 1A, Western blot analysis revealed that fascin was clearly expressed in cancer cell lines. Relatively high fascin expression was observed in highly invasive cancer cells such as

Table 1. Primer sequences, product sizes, and annealing temperatures for reverse transcription-PCR

\begin{tabular}{llc}
\hline Target gene & \multicolumn{1}{c}{ Primer sequence } & $\begin{array}{c}\text { Annealing } \\
\text { temperature } \\
\left({ }^{\circ} \mathrm{C}\right)\end{array}$ \\
\hline MMP-9 & $\begin{array}{l}\text { Sense; 5'-GATGCGTGGAGAGTCGAAAT-3' } \\
\text { Antisense; 5'-CACCAAACTGGATGACGATG-3' }\end{array}$ & 65 \\
MMP-10 & $\begin{array}{l}\text { Sense; 5'-GACCCAGACAAATGTGATCCT-3' } \\
\text { Antisense; 5'-TTCAGGCTCGGGATTCCA-3' }\end{array}$ & 60 \\
Cathepsin B & $\begin{array}{l}\text { Sense; 5'-GAAGAAGCTGTGTGGCACTG-3' } \\
\text { Antisense; 5'-GTTCGGTCAGAAATGGCTTC-3' }\end{array}$ & 58 \\
Cathepsin D & $\begin{array}{l}\text { Sense; 5'-AGGTGAAGGAGCTGCAGAAG-3' } \\
\text { Antisense; 5'-ATTCCCATGAAGCCACTCAG-3' }\end{array}$ & 58 \\
Kallikrein & $\begin{array}{l}\text { Sense; 5'-CCCCAAGTGCACTTCCCTAA-3' } \\
\text { Antisense; 5'-TCGTGTAGACACCCGGTCTG-3' }\end{array}$ & 60 \\
GAPDH & $\begin{array}{l}\text { Sense; 5'-ATCCCTCCAAAATCAAGTGGG-3' } \\
\text { Antisense; 5'-TGAAGACGCCAGTGGACTCC-3' }\end{array}$ & 60 \\
\hline
\end{tabular}

MMP, matrix metalloproteinase; GAPDH, glyceraldehyde-3-phosphate dehydrogenase.
C
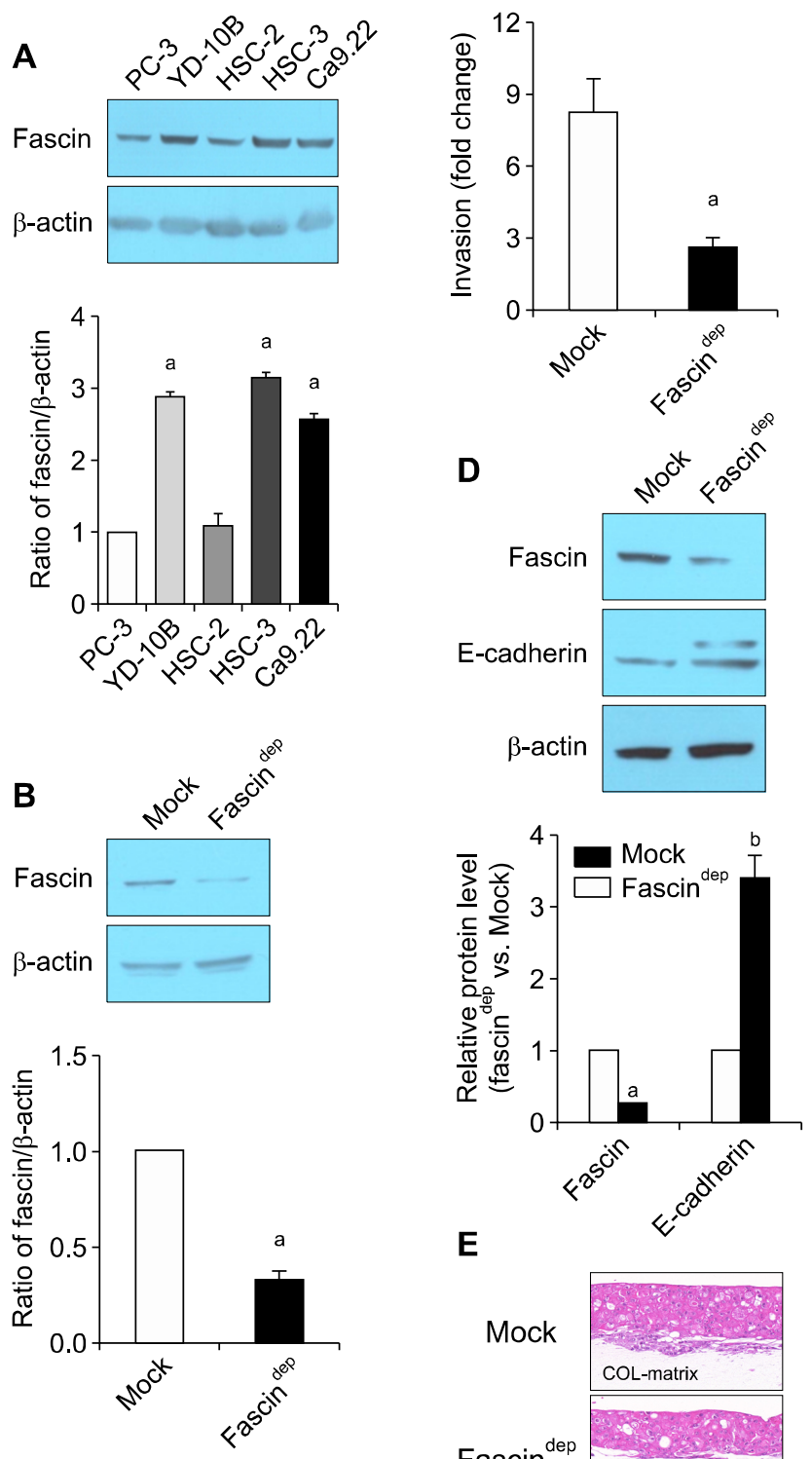

E

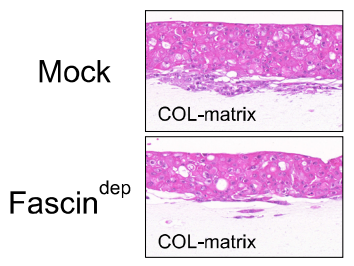

Figure 1. Effect of fascin depletion on cancer invasion. (A) Fascin expression in various cancer cells. PC-3 was loaded as fascin positive control. $\beta$-actin was used as loading control. ${ }^{a} P<0.01$ versus PC-3. (B) Fascin-depleted cell (Fascin ${ }^{\text {dep }}$ ) was prepared using fascin short hairpin RNA (shRNA) lentiviral particles and puromycine resistance clone was analyzed by Western blot for reduction of fascin level. Control shRNA lentiviral particles-A was used to prepare control stable cell line (Mock). ${ }^{a} P<0.01$ versus Mock. (C) Matrigel-coated transwell invasion activity of Mock and Fascin ${ }^{\text {dep }}$ cells. Invasion assay was proceeded for 48 hours. Invading cells were detected by hematoxylin staining and counted. ${ }^{a} P<0.01$ versus Mock. (D) E-cadherin expression in Mock and Fascin ${ }^{\text {dep }}$ cells. ${ }^{\mathrm{a}} P<0.05,{ }^{\mathrm{b}} P<0.01$ versus Mock. (E) Three-dimensional (3D) invasion of Mock and Fascin $^{\text {dep }}$ cells. Cells on Type I-A collagen mixture (COL-matrix) was culture for 10 days. Invasion of cells within dermal equivalent was analyzed by H\&E staining. Scale bars; $50 \mu \mathrm{m}$. 
A

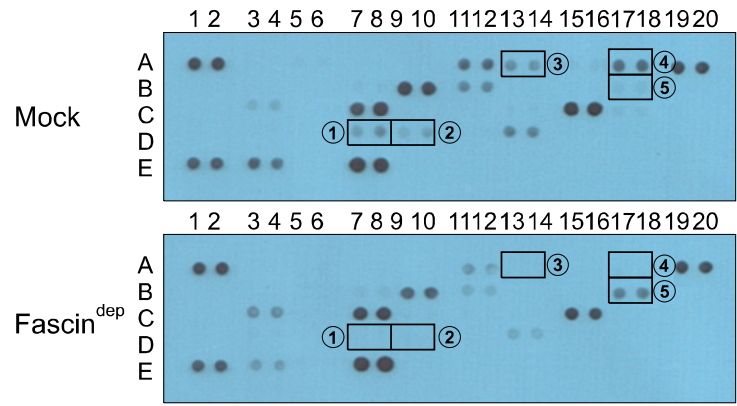

B

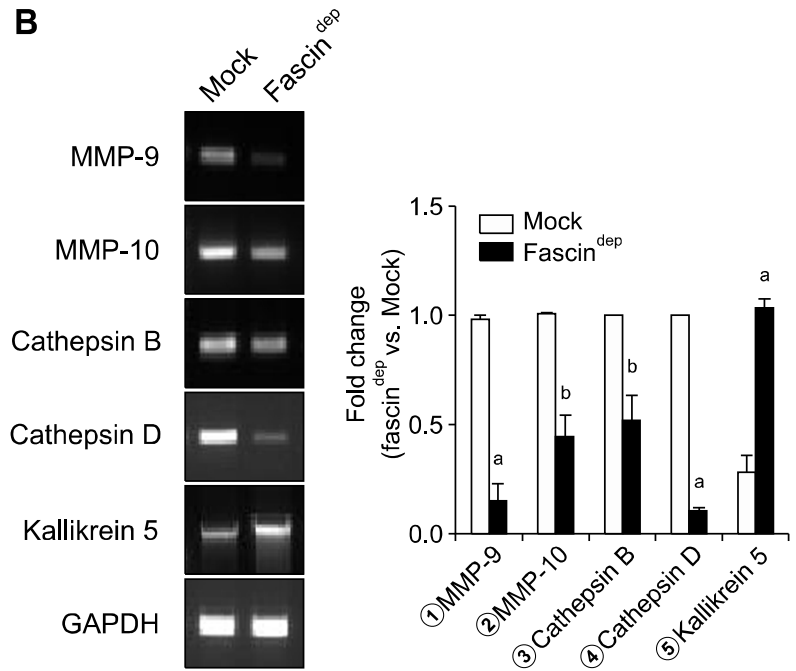

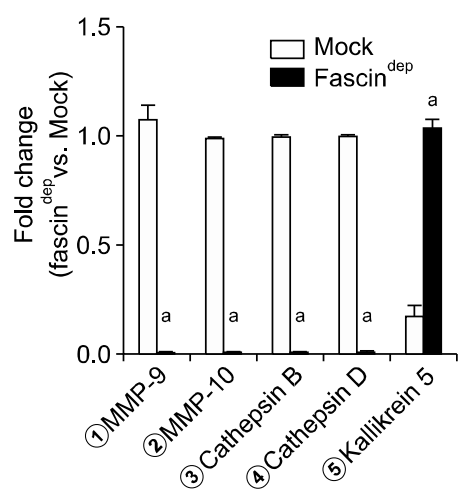$$
\text { . }
$$

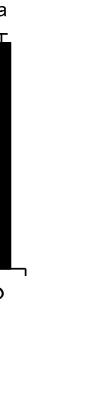

Figure 2. Effect of fascin depletion on proteases activity. (A) Released protease in Mock and Fascin ${ }^{\text {dep }}$ cells. Conditioned media from Mock and Fascin ${ }^{\text {dep }}$ cells was incubated with Protease Array Kit and relative signal intensities were determined using ImageJ software program. Results were plotted. ${ }^{a} P<0.01$ versus Mock. Proteases with large signal changes are indicated by circled numbers. (B) Expression of proteases mRNA in Mock and Fascin ${ }^{\text {dep }}$ cells. Reverse transcription-PCR products were loaded into agarose gel and glyceraldehyde-3-phosphate dehydrogenase (GAPDH) was used as RNA loading control. Results were plotted. ${ }^{\mathrm{b}} P<0.05,{ }^{\mathrm{a}} P<0.01$ versus Mock. MMP, matrix metalloproteinase.

YD-10B, HSC-3, and Ca9.22. PC-3 was analyzed as a fascin positive control. To confirm the role of fascin in cancer invasion, fascin-depleted YD-10B cell (Fascin ${ }^{\text {dep }}$ ) was established using lentiviral short hairpin (Fig. 1B). In Matrigel-coated Transwell invasion, Fascin $^{\text {dep }}$ showed significantly lower invasion activity compared to control Mock cells (Fig. 1C). The level of E-cadherin expression was lower in the control Mock, but increased in the Fascin $^{\text {dep }}$ (Fig. 1D). In addition, as a result of invasion activity, invasive areas into organotypic dermal equivalents (COL-matrix) were deeply extended in control Mock whereas Fascin ${ }^{\text {dep }}$ showed low infiltration (Fig. 1E).

\section{Effect of fascin on expression and secretion of proteases}

In order to define whether the decrease in invasion activity due to inhibition of fascin expression was caused by changes in protease activity, protease secreation was first observed. Protease array analysis showed that various protease were secreated from control Mock cells (Fig. 2A). However, the amount and type of protease secreated by fascin-depleted cells were reduced. Especially, release of matrix metalloproteinase (MMP)-9, MMP-10, cathepsin $B$, and cathepsin D were significantly decreased in Fascin ${ }^{\text {dep }}$ cells. On protease array analysis, only kallilrein 5 secreation was increased in Fascin ${ }^{\text {dep }}$ cells than that in control Mock. mRNA expression levels of MMP-9, MMP-10, cathepsin B, and cathepsin D in Fascin ${ }^{\text {dep }}$ cells were also decreased compared to those in control Mock whereas those of Kallikrein 5 (KLK5) were increased (Fig. 2B). These results indicate that fascin is associated with cancer invasion activity and that expression loss of fascin inhibits the expression and secretion of proteases, thereby decreasing cancer invasion. 


\section{DISCUSSION}

Appropriate protease secretion by cancer cells is crucial for cancer invasion activity. Cancer forms a protruding structure called invadopodia to facilitates invasion. ${ }^{6}$ When protease is secreted intensively around the invadopodia, the extracellular matrix is deeply degraded, acting as a mechanism to increase cancer cell invasion. ${ }^{4}$ Inhibition of factors involved in functional invadopodia formation such as LIMK (LIM kinase) can reduce protease activity, thereby inhibiting matrix degradation. ${ }^{7}$ Fascin is a component of invadopodia. It acts as a cross-link between actin filaments to stabilize the protruding structure of leading edges, thereby facilitating extracellular matrix invasion. ${ }^{8.9}$ In the present study, high level of fascin was expressed in a various of highly invasive cancer cells whereas fascin down-regulation significantly reduced cancer invasiveness. From this result, we analyzed proteases regulated by fascin. Despite the fact that fascin highly expressed in various cancer tissues, there is little research on the alteration of protease activity by fascin expression.

Many types of secreted proteases were detected in $\mathrm{CM}$ of oral cancer. However, several proteases were found to be limitedly secreted in fascin-depleted cell line. Secretion of MMP-9, MMP-10, cathepsin B, and cathepsin D were significantly reduced. These results were varified as mRNA expression levels of these proteases in fascin-depleted cells were also decresed. MMPs, also known as matrixins, are proteases that not only degrade all kinds of extracellular matrix proteins, but also process many bioactive molecules. They are involved in the cleavage of cell surface receptor, the release of apoptotic ligands, and chemokine activation. ${ }^{10}$ Cathepsins are also proteases found in all animals. Especially, cathepsin B is implicated in the progression of various human tumors including ovarian cancer. ${ }^{11}$ Elevated levels of cathepsin D in tumor cells have been reported to be associated with greater invasiveness. ${ }^{12}$ The reduction of cancer invasion by fascin depletion is a result of suppression of proteases transcription and secretion. However, KLK5 was increased in fascin-depleted cells in this study. KLK5 is a secreted serin protease. It cleaves various extracellular matrix components. It also activates several other KLK proteases, triggering proteolytic cascades of tissue microenvironment. Elevated level of KLK5 was detected in serum of patients with ovarian and breast cancer. ${ }^{13}$ Therefore, overexpression of KLK5 is an indicator of poor prognosis in ovarian cancer. Interestingly, KLK5 expression levels was reported to be down-regulated in malignant specimens of mammary gland compared to benign ones. ${ }^{14}$ KLK5 expression in breast tissue biopsies has been suggested as an independent biomarker for differential diagnosis between malignant tumors and benign tumors of the breast. Therefore, further clinical study is needed to compare KLK5 expression between malignant and benign oral tumors.

Epithelial-mesenchymal transition (EMT) is the process by which epithelial cells lose their cell polarity and cell-cell adhesion to gain migratory and invasive properties and become mesenchymal stem cells. ${ }^{15}$ Since EMT occurs not only in wound healing and organ fibrosis, but also in the cancer progression, cancer invasion mechanisms are understood through EMT. Epithelial cells express high levels of E-cadherin whereas mesenchymal cells express high levels of N-cadherin, fibronectin, and vimentin. Thus, EMT entails profound morphological and phenotypic changes to cell. Loss of E-cadherin is considered to be a fundamental event in EMT. E-cadherin expression is decreased in epithelial malignancies including oral cancer. It is associated with decreased cell adhesiveness and increased malignancy. Therefore, E-cadherin is a significant prognostic factor associated with relapse and survival rate. ${ }^{16}$ In this study, decreased invasion activity and increased expression of E-cadherin were observed in fascin-depleted cells. These results suggest that inhibition of fascin expression may result from suppression of EMT progression. Recent studies have indicated that invadopodia is regulated by tumor environment signals including growth factors, ${ }^{4,17}$ hypoxia, ${ }^{18} \mathrm{pH}^{19}{ }^{19}$ metabolism, ${ }^{20}$ and stromal cell interactions. ${ }^{21}$ The formation of invadopodia induced by growth factors such as epidermal growth factor, TGF- $\beta$ or platelet-derived growth factor has been reported. ${ }^{22}$ The expression of fascin induced by growth factors has also been reported. TGF- $\beta$ increases fascin expression in gastric cancer as a Smad3 phosphorylation-dependent response. ${ }^{23}$ Induction of fascin expression by interleukin- 6 and TNF- $\alpha$ in metastatic breast cancer cells has also been reported. ${ }^{24}$ These reports indicate that fascin expression and invadopodia formation are regulated by paracrine signaling pathways in the cancer microenvironment. Thus cancer invasion studies should be considered together with the tumor surrounding microenvironment.

In conclusion, fascin expression affects proteases activity for matrix degradation and regulates cancer invasion and progression. Thus, a molecular mechanism study on the regulation of the activity of proteases by fascin is important in understanding cancer invasion. Fascin may be an important target for controlling cancer invasion. 


\section{ACKNOWLEDGMENTS}

This research was supported by Basic Science Research Program through the National Research Foundation of Korea (NRF) funded by the Ministry of Education, Science and Technology (2015R1D1A1A01056946 and 2018R1D1A1B07042035).

\section{CONFLICTS OF INTEREST}

No potential conflicts of interest were disclosed.

\section{REFERENCES}

1. Guan X. Cancer metastases: challenges and opportunities. Acta Pharm Sin B 2015;5:402-18.

2. Noguti J, De Moura CF, De Jesus GP, Da Silva VH, Hossaka TA, Oshima CT, et al. Metastasis from oral cancer: an overview. Cancer Genomics Proteomics 2012;9:329-35.

3. Mehrotra R, Gupta DK. Exciting new advances in oral cancer diagnosis: avenues to early detection. Head Neck Oncol 2011;3:33.

4. Eddy RJ, Weidmann MD, Sharma VP, Condeelis JS. Tumor cell invadopodia: invasive protrusions that orchestrate metastasis. Trends Cell Biol 2017;27:595-607.

5. Hwang YS, Park KK, Chung WY. Epigallocatechin-3 gallate inhibits cancer invasion by repressing functional invadopodia formation in oral squamous cell carcinoma. Eur J Pharmacol 2013;715: 286-95.

6. Paz H, Pathak N, Yang J. Invading one step at a time: the role of invadopodia in tumor metastasis. Oncogene 2014;33:4193-202.

7. Lagoutte E, Villeneuve $C$, Lafanechère L, Wells CM, Jones GE, Chavrier $\mathrm{P}$, et al. LIMK regulates tumor-cell invasion and matrix degradation through tyrosine phosphorylation of MT1-MMP. Sci Rep 2016;6:24925.

8. Bryan J, Kane RE. Separation and interaction of the major components of sea urchin actin gel. J Mol Biol 1978;125:207-24.

9. Li A, Dawson JC, Forero-Vargas M, Spence HJ, Yu X, König I, et al. The actin-bundling protein fascin stabilizes actin in invadopodia and potentiates protrusive invasion. Curr Biol 2010;20:339-45.

10. Newby AC. Matrix metalloproteinases regulate migration, proliferation, and death of vascular smooth muscle cells by degrading matrix and non-matrix substrates. Cardiovasc Res 2006;69: 614-24.

11. Nishikawa H, Ozaki Y, Nakanishi T, Blomgren K, Tada T, Arakawa
A, et al. The role of cathepsin B and cystatin C in the mechanisms of invasion by ovarian cancer. Gynecol Oncol 2004;92: 881-6.

12. Johnson MD, Torri JA, Lippman ME, Dickson RB. The role of cathepsin D in the invasiveness of human breast cancer cells. Cancer Res 1993:53:873-7.

13. Yousef GM, Polymeris ME, Grass L, Soosaipillai A, Chan PC, Scorilas A, et al. Human kallikrein 5: a potential novel serum biomarker for breast and ovarian cancer. Cancer Res 2003;63: 3958-65.

14. Avgeris M, Papachristopoulou G, Polychronis A, Scorilas A. Down-regulation of kallikrein-related peptidase 5 (KLK5) expression in breast cancer patients: a biomarker for the differential diagnosis of breast lesions. Clin Proteomics 2011;8:5.

15. De Craene B, Berx G. Regulatory networks defining EMT during cancer initiation and progression. Nat Rev Cancer 2013;13: 97-110.

16. Shiozaki H, Tahara H, Oka H, Miyata M, Kobayashi K, Tamura S, et al. Expression of immunoreactive E-Cadherin adhesion molecules in human cancers. Am J Pathol 1991;139:17-23.

17. Gould CM, Courtneidge SA. Regulation of invadopodia by the tumor microenvironment. Cell Adh Migr 2014:8:226-35.

18. Lucien F, Brochu-Gaudreau K, Arsenault D, Harper K, Dubois CM. Hypoxia-induced invadopodia formation involves activation of NHE-1 by the p90 ribosomal S6 kinase (p90RSK). PLoS One 2011; 6:e28851.

19. Rosenthal CK. pH-dependent invadopodia control. Nature Cell Biology 2012;14:50.

20. van Horssen R, Buccione R, Willemse M, Cingir S, Wieringa B, Attanasio F. Cancer cell metabolism regulates extracellular matrix degradation by invadopodia. Eur J Cell Biol 2013;92:113-21.

21. Cao H, Eppinga RD, Razidlo GL, Krueger EW, Chen J, Qiang L, et al. Stromal fibroblasts facilitate cancer cell invasion by a novel invadopodia-independent matrix degradation process. Oncogene 2016:35:1099-110.

22. Murphy DA, Courtneidge SA. The 'ins' and 'outs' of podosomes and invadopodia: characteristics, formation and function. Nat Rev Mol Cell Biol 2011;12:413-26.

23. Li L, Cao F, Liu B, Luo X, Ma X, Hu Z. TGF- $\beta$ induces fascin expression in gastric cancer via phosphorylation of smad3 linker area. Am J Cancer Res 2015:5:1890-6.

24. Snyder M, Huang J, Huang XY, Zhang JJ. A signal transducer and activator of transcription 3.nuclear factor $\kappa \mathrm{B}(\mathrm{Stat} 3 \cdot \mathrm{NF} \kappa \mathrm{B})$ complex is necessary for the expression of fascin in metastatic breast cancer cells in response to interleukin (IL)-6 and tumor necrosis factor (TNF)- $\alpha$. J Biol Chem 2014:289:30082-9. 